\title{
EMPRENDIMIENTO PARA LA CARRERA DE EDUCACIÓN INICIAL
}

\section{ENTREPRENEURSHIP TO EARLY CHILDHOOD EDUCATION CAREERS}

\author{
Erika Margarita Pazmiño Cárdenas ${ }^{1}$ \\ Ivonne Augusta Andino Sosa ${ }^{2}$ \\ Karla Fabiola Romero Coronel ${ }^{3}$ \\ María Fernanda Becerra Sarmiento 4 \\ Ivonne Anabel Pazmiño Cárdenas ${ }^{5}$
}

Recibido: 2021-05-10 / Revisado: 2021-07-10 / Aceptado: 2021-08-12 / Publicado: 2021-09-15

Forma sugerida de citar: Pazmiño-Cárdenas, E. M., Andino-Sosa, I. A., Romero-Coronel, K. F., Becerra-Sarmiento, M. F. y Pazmiño-Cárdenas, I. A. (2021). Emprendimiento para la Carrera de Educación Inicial. Retos de la Ciencia. 5(e). 52-63. https://doi.org/10.53877/rc.5.e.20210915.05

\section{RESUMEN}

A partir de la pandemia COVID-19 declarada por la Organización Mundial de la Salud, el ámbito laboral en el sector educativo fue afectado, sobre todo en el nivel inicial por la suspensión de las clases presenciales, el ingreso a la educación virtual y en ciertos casos, el cierre de instituciones educativas. Desde esta perspectiva, el estudio tiene la finalidad de describir la formación docente universitaria en el campo del emprendimiento en la Carrera de Educación Inicial de las Universidades públicas y privadas de Ecuador. La información fue recolectada a través un amplio análisis epistemológico, así como también, de las mallas curriculares, el perfil de egreso y el campo laboral proyectado de la carrera que guardan relación con el emprendimiento como parte de la formación académica. Los resultados revelan que de las 30 universidades que ofertan la referida carrera; 13, contemplan en su malla curricular la asignatura de Emprendimiento, 6, la relacionan con su campo laboral proyectado y; 4

1 Magíster en Educación Parvularia. Docente Investigador de la Universidad Tecnológica Indoamérica. Ecuador. E-mail: epazmino3@indoamerica.edu.ec / ORCID: https://orcid.org/0000-0002-0441-2710

2 Magíster en Desarrollo Educativo. Docente Investigador de la Universidad Tecnológica Indoamérica. Ecuador. E-mail: iandino@indoamerica.edu.ec / ORCID: https://orcid.org/0000-0003-3034-9855

3 Magíster en Educación y Desarrollo del Pensamiento. Docente Investigador de la Universidad Tecnológica Indoamérica. Ecuador. E-mail: kromero2@indoamerica.edu.ec / ORCID: https://orcid.org/0000-0002-9224-227 4 Magíster en Gestión del Talento Humano. Docente Investigador de la Universidad Tecnológica Indoamérica. Ecuador. E-mail: mariabecerra@uti.edu.ec / ORCID: https://orcid.org/0000-0002-8599-8935

5 Magíster en Docencia Universitaria y Administración Educativa. Docente Investigador de la Pontificia Universidad Católica del Ecuador. Ecuador. E-mail: ipazmino176@puce.edu.ec / ORCID: https://orcid.org/0000-0002-51114476 
la consideran en su perfil de egreso. Lo cual evidencia, la limitada consideración al emprendimiento en la formación de profesionales de educación inicial, distanciándose, de las reales necesidades del campo laboral vertiginosamente cambiante en la actualidad. y lo empresarial que se constituiría como una posible solución a las necesidades sociales y laborales actuales. A futuro es necesario la revisión de los syllabus de esta asignatura para verificar el desarrollo de competencias emprendedoras.

Palabras claves: carrera de Educación Inicial, campo laboral, emprendimiento, malla curricular, perfil de egreso.

\section{ABSTRACT}

Since the COVID-19 pandemic declared by the World Health Organization, the work environment in the education sector has been affected, especially at the early education level, due to the suspension of face-to-face classes, the introduction of virtual education and, in some cases, the closure of educational institutions. From this perspective, the purpose of this study is to describe university teacher training in the field of entrepreneurship in the Early Childhood Education program at public and private universities in Ecuador. The information was collected through a broad epistemological analysis, as well as the curricula, the graduate profile and the projected labor field of the career that are related to entrepreneurship as part of academic training. The results reveal that of the 30 universities that offer this career, 13 include entrepreneurship as a subject in their curricula, 6 relate it to their projected field of work and 4 include it in their graduate profile. This shows the limited consideration of entrepreneurship in the training of initial education professionals, which distances them from the real needs of today's rapidly changing labor field. In the future, it is necessary to review the syllabus of this subject to verify the development of entrepreneurial competencies.

Keywords: early childhood education career; labor field; entrepreneurship; curriculum; graduate profile.

\section{INTRODUCCIÓN}

La declaración de la Organización Mundial de la Salud de la pandemia por COVID-19 en el año 2020 modificó la dinámica de las sociedades, muchos sectores fueron afectados severamente por las medidas de confinamiento y paralización de actividades como es el caso de la economía, educación, política, salud, turismo, entre otros. Al igual que en otras pandemias pandemias que generaron consecuencias graves, se prevé de acuerdo con el Banco Mundial (2021) que los efectos adversos serán de larga duración a nivel mundial por la falta de inversión, cierre de empresas y fábricas, el desempleo y subempleo.

El sistema educativo fue uno de los sectores más afectados a nivel mundial, ya que, no estaba lo suficientemente preparado para una disrupción educativa que obligó al cambio de la modalidad de los estudios presenciales a la virtualidad. La Organización de las Naciones Unidas para la Educación, la Ciencia y la Cultura [ONU] (2020), señala que esta medida fue establecida para garantizar la continuidad de la educación. Esto dio lugar al cierre de varias instituciones, como se señala por el Banco Mundial (2021), caracterizada por la reducción de la oferta y demanda 
educativa, deserción escolar, evidenciándose la mayor desigualdad y crisis económica en las diferentes regiones.

De acuerdo con la Comisión Económica para América Latina y el Caribe (CEPAL), en el año 2020, antes de presentarse la pandemia; América Latina ya enfrentaba una gran brecha social, con índices de pobreza y extrema pobreza elevados, inequidad, desigualdad y descontento social. En el campo educativo, la realidad no difiere, las clases fueron suspendidas en todos los niveles, pasando de la presencialidad a la virtualidad, diversificando las modales de aprendizaje desde el uso de plataformas para quienes tenían acceso a tecnología hasta el desplazamiento del personal educativo a comunidades que no contaban con la misma.

A un año de los cambios generados por la pandemia en las naciones, millones de niños, adolescentes y jóvenes han enfrentado el cierre parcial o definitivo de algunas instituciones educativas, afectando su proceso de enseñanza aprendizaje; además, dicha afectación alcanzó a los docentes por la inestabilidad laboral existente incrementándose el desempleo en especial en la educación Inicial que atiende a menores de seis años.

Para mitigar este impacto en el ámbito laboral, el sistema de educación superior que forma a profesionales en docencia debe incorporar en sus carreras un enfoque basado en la educación emprendedora y empresarial que contribuirá con la construcción de conocimientos y el desarrollo de habilidades, actitudes y valores, así como, la adquisición de herramientas necesarias para transformar su realidad en beneficio personal y comunitario a partir de sus potencialidades. De acuerdo a ArayaPizarro y Avilés-Pizarro (2019), emprender a más de generar réditos económicos, desarrolla el ámbito personal, bienestar emocional, habilidades y competencias para insertarse en los nuevos mercados laborales.

La cultura emprendedora generada en países de alto desarrollo surge como resultado de la incorporación de procesos de aprendizaje enfocados en la formación de habilidades y valores para potencializar las aptitudes y patrones de comportamiento en los individuos direccionadas a emprender. Johannisson (2016) señala que en Suecia existen maneras radicalmente diferentes de formación académica para el emprendimiento, que dejan a un lado la ambigüedad y aplican conocimiento científico que generan un impacto, especialmente en las competencias empresariales que requiere el estudiante al gestionar un emprendimiento. La mejora continua de la academia implica que las universidades intervengan y provoquen un clima favorable e inspirador en el estudiante para convertirse en empresario, como bien lo señala Bergmann, et al. (2018), ya que al adquirir competencias emprendedores y empresariales vinculadas al sector laboral productivo responderán a las necesidades cambiantes de la sociedad de manera proactiva para enfrenter los problemas del contexto actual.

Los emprendimientos fortalecen la economía local, nacional y regional al generar ingresos propios, nuevas oportunidades y fuentes de empleo. Desde esta perspectiva, las universidades que promueven la cultura emprendedora en la formación académica generan un impacto positivo en la actitud de los estudiantes. Jena (2020), señala que los profesionales relacionados con el emprendimiento durante su proceso de formación lo consideran, como una oportunidad empresarial para su independencia laboral. Por lo tanto, las universidades deben generar espacios de tal manera que, la practicidad del conocimiento, las ideas de ideas de negocio que fueron parte del estudio en las aulas de clase sean materializadas el campo laboral como lo señalan Huq y Gilbert (2017). 
Esto se logra, desde la transversalidad del Emprendimiento en todos los ámbitos de formación: vinculación, investigación y prácticas pre-profesionales. Además, Alvarado y Martínez (2011), señala que, la implantación de un modelo curricular que establezca espacios, contenidos, estrategias que faciliten la creación, potenciación y desarrollo de ideas innovadoras, es decir, generen incubadoras de ideas, garantizarán resultados en la creación de emprendimientos por parte de los profesionales.

Por lo tanto, las instituciones de educación superior con el apoyo de entidades gubernamentales y no gubernamentales deben promover la inserción de proyectos empresariales en sus mallas curriculares para ejecutar en la praxis todos los conocimientos impartidos en las aulas de clase, desarrollando iniciativas que guarden relación con su campo de formación proyectado. Es decir, de acuerdo con Martínez et al (2016) el aula de clases debe convertirte en incubadoras de pensamientos nuevos como un medio de solución a las necesidades del entorno.

El impacto esperado al implementar estrategias emprendedoras en el aula de clase no solo debe estar orientado a formar estudiantes propositivos y emprendedores. Este impacto debe permitir que los docentes se empoderen de la dinámica emprendedora para la mejora del proceso enseñanza aprendizaje. Desde esta perspectiva, los docentes que imparten la cátedra de emprendimiento también requieren de competencias técnicas y conductuales para motivar la práctica y ejecución de los emprendimientos, dentro y fuera de las aulas de clase. Utami (2017) señala que la actitud tanto del docente como del estudiante constituyen factores primordiales a la hora de emprender, ya que transforman las intenciones y la visión empresarial como una fuente económica de generación de empleo.

El emprendimiento en el Ecuador ha alcanzado un impacto positivo, por lo que, ha sido objeto de estudio de investigadores, gobierno y sectores privados (Morales, et al., 2020) considerándose una apuesta para el desarrollo socioeconómico del país, reactivando la misma. Por lo tanto, es necesario promover el interés hacia el emprendimiento en las aulas de clase de las universidades ecuatorianas mediante programas educativos que impulsen el desarrollo de competencias que caracterizan a los emprendedores, como lo hacen en la formación académica de los profesionales de los países desarrollados. Estas competencias emprendedoras de acuerdo a Valencia, et al. (2021) se evidencian en las relaciones interpersonales, trabajo en equipo, orientación de servicio, liderazgo, creatividad e innovación. De ahí que cabe preguntarse ¿qué universidades públicas y privadas del Ecuador contemplan la asignatura de emprendimiento en su malla de la Carrera de Educación Inicial? y ¿qué universidades contemplan dentro del perfil de salida y campo laboral proyectado la relación con el emprendimiento?

A partir de la presente investigación se plantea como objetivo el identificar la relación de la Carrera de Educación Inicial de las Universidades del Ecuador con su formación en Emprendimiento mediante la revisión de la malla curricular, el perfil y campo laboral proyectado que ofertan.

\section{METODOLOGÍA}

Este estudio es de enfoque cualitativo, porque caracteriza al objeto de estudio como un hecho social como es el emprendimiento en la formación del profesorado en educación inicial en función de las condiciones del contexto. Además, se remite a la revisión teórica en profundidad de fuentes publicadas en medios de alto impacto. 
Para alcanzar el objetivo planteado se realizó una búsqueda minuciosa de las páginas web de universidades públicas y privadas del Ecuador que ofertan la Carrera de Educación Inicial de acuerdo con los criterios de inclusión que se describen a continuación:

a. Que las universidades indagadas contemplan la carrera de Educación Inicial ya sena públicas o privadas.

b. Que las universidades indagadas oferten sus mallas curriculares la asignatura de Emprendimiento para la formación del profesorado en la Carrera de Educación Inicial.

c. Que las universidades indagadas señalen en su perfil de egreso una relación con el Emprendimiento; y,

d. Que las universidades indagadas describan al emprendimiento en su campo laboral proyectado de la carrera (demanda)

Se excluyeron las universidades públicas y privadas que no cumplían con estos cuatro criterios. Con esta búsqueda y revisión de las diversas fuentes se propuso responder a las interrogantes de investigación.

\section{Gráfico 1:}

Esquema de búsqueda de información

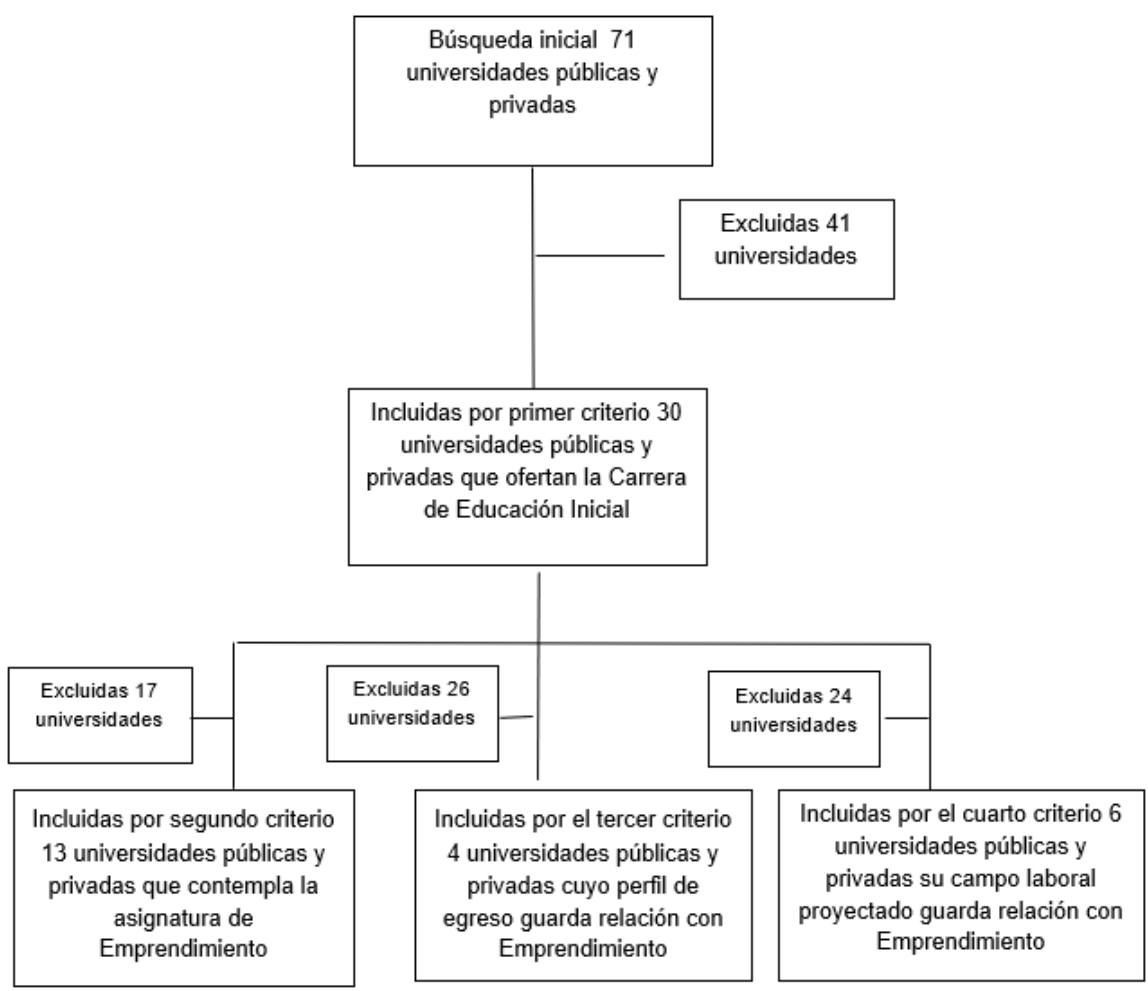

Elaborado por: elaboración propia

\section{RESULTADOS}

Se puede observar que en el Ecuador existen 71 universidades entre públicas y privadas de las cuales solo 30 ofertan la Carrera de Educación Inicial, distribuidas por el sostenimiento ya sea privado (12 instituciones) y público (18 instituciones); 9 particulares y 10 fiscales en la región Sierra; 4 particulares y 8 fiscales en la región 
Costa, evidenciando una mayor oferta de dicha carrera en instituciones con sostenimiento privado en la región Sierra (Ver Tabla 1).

Tabla 1:

Universidades en el Ecuador que ofertan la Carrera de Educación Inicial

\begin{tabular}{llll}
\hline No. & UNIVERSIDAD & SOSTENIMIENTO & REGIÓN \\
\hline 1 & Universidad Católica de Cuenca & Particular & Sierra \\
2 & Universidad del Azuay & Particular & Sierra \\
3 & Universidad Politécnica Salesiana & Particular & Sierra \\
4 & Universidad Metropolitana & Particular & Sierra \\
5 & Universidad Tecnológica Indoamérica & Particular & Sierra \\
6 & Universidad Técnica Particular de Loja & Particular & Sierra \\
7 & Universidad Tecnológica Israel & Particular & Sierra \\
8 & Pontificia Universidad Católica del Ecuador & Particular & Sierra \\
9 & Universidad Católica del Ecuador & Particular & Sierra- \\
10 & Universidad Casa Grande & Particular & Costa \\
11 & Universidad Particular de Especialidades & Particular & Costa \\
12 & Universidad Particular San Gregorio de & Particular & Costa \\
13 & Universidad de Cuenca & Fiscal & Sierra \\
14 & Universidad Estatal de Bolívar & Fiscal & Sierra \\
15 & Universidad Nacional de Educación & Fiscal & Sierra \\
16 & Universidad Nacional de Chimborazo & Fiscal & Sierra \\
17 & Universidad Técnica de Cotopaxi & Fiscal & Sierra \\
18 & Universidad Técnica del Norte & Fiscal & Sierra \\
19 & Universidad Nacional de Loja & Fiscal & Sierra \\
20 & Universidad Central del Ecuador & Fiscal & Sierra \\
21 & Universidad de las Fuerzas Armadas - ESPE & Fiscal & Sierra \\
22 & Universidad Técnica de Ambato & Fiscal & Sierra \\
23 & Universidad Técnica de Machala & Fiscal & Costa \\
24 & Universidad Técnica Luis Vargas Torres & Fiscal & Costa \\
25 & Universidad de Guayaquil & Fiscal & Costa \\
26 & Universidad Estatal de Milagro & Fiscal & Costa \\
27 & Universidad Técnica de Babahoyo & Fiscal & Costa \\
28 & Universidad Laica Eloy Alfaro de Manabí & Fiscal & Costa \\
29 & Universidad Técnica de Manabí & Fiscal & Costa \\
30 & Universidad Estatal Península de Santa Elena & Fiscal & Cinavacionn
\end{tabular}

Nota. Tomado de Secretaría de Educación Superior, Ciencia, Tecnología e Innovación (SENESCYT), listado de universidades del Ecuador de acuerdo con el lugar geográfico.

Se enlistan 13 universidades de la región Costa y Sierra que ofertan la Carrera de Educación Inicial y consideran en su malla curricular la asignatura de Emprendimiento. Como se observa la nominación varía, sin embargo, todas están direccionadas a dicha asignatura (Ver tabla 2). 
Tabla 2:

Universidades en el Ecuador que oferta en la Carrera de Educación Inicial la Asignatura de Emprendimiento

\begin{tabular}{|c|c|c|c|}
\hline No. & UNIVERSIDAD & ASIGNATURA & REGIÓN \\
\hline 1 & $\begin{array}{lll}\text { Universidad } & \text { Católica } & \text { de } \\
\text { Cuenca } & & \end{array}$ & $\begin{array}{l}\text { Innovación y emprendimiento } \\
\text { educativo }\end{array}$ & Sierra \\
\hline 2 & $\begin{array}{l}\text { Universidad } \\
\text { Indoamérica }\end{array}$ & Liderazgo y emprendimiento & Sierra \\
\hline 3 & $\begin{array}{ll}\text { Universidad } & \text { Técnica } \\
\text { Particular de Loja } & \end{array}$ & Emprendedurismo & Sierra \\
\hline 4 & $\begin{array}{l}\text { Universidad Tecnológica } \\
\text { Israel }\end{array}$ & Emprendimiento e innovación & Sierra \\
\hline 5 & Universidad de Cuenca & Gestión y Liderazgo & Sierra \\
\hline 6 & $\begin{array}{l}\text { Universidad Técnica de } \\
\text { Cotopaxi }\end{array}$ & Emprendimiento Social & Sierra \\
\hline 7 & $\begin{array}{l}\text { Universidad de las Fuerzas } \\
\text { Armadas - ESPE }\end{array}$ & $\begin{array}{l}\text { Gestión de la innovación y el } \\
\text { emprendimiento }\end{array}$ & Sierra \\
\hline 8 & $\begin{array}{l}\text { Universidad Técnica de } \\
\text { Ambato }\end{array}$ & $\begin{array}{l}\text { Gestión de Proyectos Socio } \\
\text { productivos }\end{array}$ & Sierra \\
\hline 9 & $\begin{array}{l}\text { Universidad Particular de } \\
\text { Especialidades Espíritu Santo }\end{array}$ & Liderazgo emprendedor & Costa \\
\hline 10 & $\begin{array}{l}\text { Universidad Particular San } \\
\text { Gregorio de Portoviejo }\end{array}$ & Emprendedurismo & Costa \\
\hline 11 & $\begin{array}{l}\text { Universidad Estatal de } \\
\text { Milagro }\end{array}$ & Emprendimiento e Innovación & Costa \\
\hline 12 & $\begin{array}{l}\text { Universidad Técnica de } \\
\text { Manabí }\end{array}$ & $\begin{array}{l}\text { Emprendimiento e Innovación } \\
\text { Profesional }\end{array}$ & Costa \\
\hline 13 & $\begin{array}{l}\text { Universidad Estatal Península } \\
\text { de Santa Elena }\end{array}$ & $\begin{array}{l}\text { Emprendimiento e Innovación } \\
\text { Educativa }\end{array}$ & Costa \\
\hline
\end{tabular}

Nota: Adaptado de mallas curriculares de las Universidades del Ecuador

Los niveles de formación en que se dicta la asignatura de Emprendimiento de las 13 universidades que ofertan la Carrera de Educación Inicial, dan como resultado que hay 3 instituciones en el nivel básico; 3 en el nivel profesional y 7 en el nivel de titulación. A partir de los resultados se evidencia una tendencia a impartir la asignatura en los últimos niveles de la formación del docente de educación inicial (Ver tabla 3).

Tabla 3:

Semestre en el que se dicta la asignatura de Emprendimiento

\begin{tabular}{|c|c|c|c|}
\hline \multirow[b]{2}{*}{ Universidad } & \multicolumn{2}{|c|}{ Niveles de Formación } & \multirow[b]{2}{*}{ Titulación } \\
\hline & Básico & Profesionalización & \\
\hline Universidad Católica de Cuenca & & & $8 \mathrm{vo}$ \\
\hline Universidad Tecnológica Indoamérica & & $7 \mathrm{mo}$ & \\
\hline Universidad Técnica Particular de Loja & & & 8vo \\
\hline Universidad Tecnológica Israel & $2 \mathrm{dc}$ & & \\
\hline Universidad de Cuenca & & & $8 \mathrm{vo}$ \\
\hline Universidad Técnica de Cotopaxi & $3 e r$ & & \\
\hline $\begin{array}{l}\text { Universidad de las Fuerzas Armadas - } \\
\text { ESPE }\end{array}$ & & 6 to & \\
\hline Universidad Técnica de Ambato & & & 9 no \\
\hline $\begin{array}{l}\text { Universidad Particular de Especialidades } \\
\text { Espíritu Santo }\end{array}$ & 1ro & & \\
\hline $\begin{array}{l}\text { Universidad Particular San Gregorio de } \\
\text { Portoviejo }\end{array}$ & & 6 to & \\
\hline Universidad Estatal de Milagro & & & 8vo \\
\hline Universidad Técnica de Manabí & & & $8 \mathrm{vo}$ \\
\hline
\end{tabular}


Elena

Nota: Adaptado de mallas curriculares de las Universidades del Ecuador

Existen 4 universidades que describen en el perfil de egreso una relación con la asignatura de Emprendimiento, evidenciándose que estas instituciones están orientando la formación de sus profesionales hacia el emprendimiento como agentes de transformación social (Ver tabla 4).

Tabla 4:

Perfil de egreso que guarda relación con emprendimiento

\begin{tabular}{|c|c|c|}
\hline Universidades & Región & Perfil de egreso \\
\hline $\begin{array}{l}\text { Universidad Técnica Luis } \\
\text { Vargas Torres }\end{array}$ & Costa & Capacidad de emprendimiento. \\
\hline $\begin{array}{l}\text { Universidad Particular San } \\
\text { Gregorio de Portoviejo }\end{array}$ & Costa & $\begin{array}{l}\text { Profesional con sólida formación teórica, metodológica, } \\
\text { investigativa, tecnológica e instrumental en los ámbitos } \\
\text { del desarrollo y aprendizajes del nivel inicial, con manejo } \\
\text { adecuado de la interdisciplinariedad, para dar respuesta } \\
\text { asertiva a los problemas educativos, desde los enfoques } \\
\text { humanístico, intergeneracional, inclusivo e intercultural, } \\
\text { encaminado a la satisfacción de su proyecto de vida y la } \\
\text { generación de emprendizajes innovadores. }\end{array}$ \\
\hline $\begin{array}{l}\text { Universidad Central del } \\
\text { Ecuador }\end{array}$ & Sierra & $\begin{array}{l}\text { Los resultados de la gestión de escenarios profesionales } \\
\text { que implican los emprendizajes en la práctica social de } \\
\text { los futuros profesionales. }\end{array}$ \\
\hline Universidad Metropolitana & Sierra & $\begin{array}{l}\text { Mejora Continua: Contribuirá al crecimiento personal, } \\
\text { empresarial y de la colectividad ecuatoriana, al trabajar } \\
\text { en equipo y de forma autónoma y diseñar programas de } \\
\text { atención a la educación inicial para mejorar la calidad de } \\
\text { vida. }\end{array}$ \\
\hline
\end{tabular}

Nota: Adaptado de páginas web oficiales de las universidades

Se evidencian 6 universidades que establecen una relación entre el campo laboral y el enfoque de emprendimiento en la Carrera de Educación Inicial. Esto muestra una tendencia en el campo laboral hacia la asesoría pedagógica, proyectos educativos y apertura de centros infantiles (Ver tabla 5).

Tabla 5:

Campo laboral que guarda relación con emprendimiento

\begin{tabular}{|c|c|c|}
\hline Universidad & Región & Campo laboral \\
\hline Universidad Casa Grande & Costa & $\begin{array}{l}\text { Brindar asesoramiento pedagógico a familias, } \\
\text { instituciones y docentes para implementar programas, } \\
\text { proyectos e intervenciones educativas innovadoras. } \\
\text { Emprendimientos y proyectos. }\end{array}$ \\
\hline $\begin{array}{l}\text { Universidad Particular de } \\
\text { Especialidades Espíritu } \\
\text { Santo }\end{array}$ & Costa & $\begin{array}{l}\text { Evaluación y asesoría educativa/ psicopedagógica } \\
\text { educación inclusiva y especial. Industrias de material } \\
\text { didáctico para niños. }\end{array}$ \\
\hline $\begin{array}{l}\text { Universidad Técnica de } \\
\text { Babahoyo }\end{array}$ & Costa & $\begin{array}{l}\text { Experimenta, desarrolla, innova, investigaciones } \\
\text { aplicadas en el área acordes a las necesidades del país. }\end{array}$ \\
\hline $\begin{array}{l}\text { Universidad Laica Eloy } \\
\text { Alfaro de Manabí }\end{array}$ & Costa & $\begin{array}{l}\text { Docente independiente aplicando proyectos de } \\
\text { investigación pedagógica. Administra y organiza centros } \\
\text { infantiles propios o privados. }\end{array}$ \\
\hline $\begin{array}{l}\text { Universidad de las } \\
\text { Fuerzas Armadas - ESPE }\end{array}$ & Sierra & $\begin{array}{l}\text { Emprendimiento. Demanda de competencias en } \\
\text { educación inicial como asesoría para material didáctico, } \\
\text { libros educativos, ambientes de aprendizaje. }\end{array}$ \\
\hline
\end{tabular}


Universidad Tecnológica Sierra Director/a, consultor/a o promotor/a de una empresa Israel educativa.

Nota: Adaptado de páginas web oficiales de las universidades

\section{DISCUSIÓN}

Los cambios vertiginosos que experimenta el mundo son excepcionales, la pandemia de la COVID-19 ha transformado muchos ámbitos evidenciando cuán preparado está el ser humano para enfrentar las problemáticas que surgen en los diferentes sectores. La universidad debe estar alineada a las tranformaciones, los cambios y necesidades de desarrollo social, apuntalando al desarrollo de capacidades, conocimientos y habilidades que les permitan a los estudiantes ser generadores de emprendimientos como formas de sostenimiento. En concordancia con los datos expuestos, se observa que, las universidades ecuatorianas públicas y privadas, consideran muy limitadamente la asignatura del Emprendimiento dentro de la malla curricular para la formación de profesores en la Carrera de Educación Incial, lo cual se sustenta en lo siguiente:

De las 30 universidades que tienen la Carrera de Educación Inicial en el Ecuador tanto en la región Sierra como Costa, la asignatura de Emprendimiento es considerada únicamente por 13 instituciones de educación superior; mientras que aquellas que la relacionan con su campo laboral proyectado apenas son 6 y tan solo 4, la consideran en su perfil de egreso. Los resultados muestran que la formación académica de la cultura de emprendimiento se aborda por un mínimo número de instituciones de educación superior, evidenciando que la sociedad no la potencia y que las políticas sociales que favorecen el emprendimiento son escasas. (Paternina, 2018)

Las instituciones de educación superior que contemplan la asignatura de Emprendimiento la ofertan en su malla bajo las denominaciones relacionadas con liderazgo, proyectos e innovación, con una serie de contenidos relacionados y a la vez, direccionados hacia varios ámbitos, sin centrarse, en el emprendimiento como tal. En ocasiones estas asignaturas responden a las perspectivas teóricometodológicas que sustentan la carrera y la formación profesional de los estudiantesorientado a generar una dependencia laboral como un mecanismo de sustentación económica

Las asignaturas relacionadas con el emprendimiento se imparten en los diferentes niveles de formación, siendo más notorio en el de titulación, de aquí nacen interrogantes, ¿qué beneficio tiene el impartir esta asignatura cuando el estudiante está culminando su formación profesional? o ¿cuál es el nivel de formación más apropiado para cursar esta asignatura? El Ministerio de Educación (2011) en la Ley Orgánica de Educación Intercultural, establece que el Bachillerato Técnico debe promover como parte de la formación complementaria los estudiantes, el ingresar al mercado laboral e iniciar actividades de emprendimiento social o económico. Esto es importante considerarlo, ya que las instituciones de superior deben procurar la articulación de este aspecto en el diseño de las carreras para promover la continuidad en la formación emprendedora de los estudiantes.

En cuanto al perfil de egreso, se describe las competencias emprendedoras de manera general, señalando que los emprendizajes pueden ser innovadores aplicados a la práctica social, como aporte a una mejora continua al crecimiento personal y empresarial. A partir de esta información, no se evidencia con certeza la formación de profesores emprendedores de la Carrera de Educación Incial que busquen 
alternativas para sustentarse económicamente cuando su plaza de trabajo sea afectada, o considerar los enfoques didácticos que aplican para obtener el impacto deseado como le señala Maresch, et al. (2016)

En cuanto al campo laboral proyectado se direcciona el emprendimiento al asesoramiento y evaluación de centros infantiles, proyectos de intervención, industria de material didáctico, apertura de centros educativos y elaboración de proyectos educativos. Los ámbitos en que los docentes emprendedores pueden incursionar son amplios; sin embargo, no son considerados en el campo laboral planteado por las instituciones de educación superior, limitando su accionar y desempeño laboral para mejorar su condición de vida. Desde la academia es importante abordar políticas institucionales para ofertar el emprendimiento en su perfil de salida y campo laboral del futuro educador implementando convenios de cooperación interinstitucional, generando estrategias y recursos materiales, tecnológicos y financieros que potencien la concreción de las ideas innovadoras de emprendimiento (Vásquez, 2017)

El rol que juegan las instituciones de educación superior es imprescindible en la formación del futuro docente, ya que deben responder a las necesidades actuales de la sociedad. La implementación del emprendimiento como un eje transversal es primordial para posibilitar la formación de profesionales visionarios y empresarios competentes para el desarrollo socio-productivo y la mejora de la calidad de vida. La generación de espacios en las instituciones de educación superior en donde, el aprendizaje sea más práctico es fundamental para la generación de la cultura emprendedora, así como la implementación de programas que aborden la formación de capacidades emprendedoras en los estudiantes. Pero a su vez, los docentes constituyen un pilar importante en la motivación a los estudiantes, direccionada a la generación de actividades rentables que mejoren su calidad de vida y enfrenten los desafíos actuales. La motivación emprendedora (Barba yAtienza, 2018) depende de la capacidad del docente sin importar la carrera, para transformar sus horas de clase en experiencias únicas, que lleven a un nivel que los estudiantes intuyan y entiendan la diferencia entre generar empleo y ser empleado.

\section{REFERENCIAS}

Alvarado, O., \& Martinez, W. (22 de junio de 2011). Universidad y emprendimiento, aportes para la formación de profesionales emprendedores. Red de Revistas Científicas de América Latina, el Caribe, España y Portugal (Redalyc.org). https://www.redalyc.org/pdf/2250/225019868005.pdf

Araya-Pizarro, S., \& Avilés-Pizarro, N. (29 de junio de 2019). Enseñar a emprender en universidades de la Región de Coquimbo, Chile: Perfil docente y prácticas de enseñanza. Redalyc.org. https://www.redalyc.org/jatsRepo/447/44764873002/index.html

Banco Mundial. (5 de enero de 2021). Banco Mundial. Recuperado el 30 de abril 2021 de https://acortar.link/NvJXD

Bergmann, H., Geissler, M., Hundt, C., \& Grave, B. (2018). The climate for entrepreneurship at higher education institutions. Research Policy. 47 (4), 700 716. https://doi.org/10.1016/j.respol.2018.01.018

Comisión Económica para América Latina y el Caribe (CEPAL). (13 de agosto de 2020). Naciones Unidas. Recuperado el 30 de abril de 2020, de https://repositorio.cepal.org/handle/11362/45904 
Entrialgo,M.\& Iglesias,V. (2016). The moderating role of entrepreneurship education on the antecedents of entrepreneurial intention. International Entrepreneurshi $p$ and Management Journal. 12

(4), 1209-1232. https://doi.org/10.1007/s11365-016-0389-4

Fayolle,

A., Verzat,

C.,

\& Wapshott, $R$. (2016). In quest of legitimacy: The theoretical and methodological foundations of entrepreneurship education research. International Small

Business Journal: Researching Entrepreneurship. 34

895-904. https://doi.org/10.1177/0266242616649250

Huq, A., Gilbert, D. (2017). All the world's a stage: transforming entrepreneurship education through design thinking. Education and Training. 59 (2), 155-170 https://doi.org/10.1108/ET-12-2015-0111

Instituto Nacional de Evaluación Educativa (INEVAL). (2017-2018). La educación en Ecuador: Logros alcanzados y nuevos desafíos. Ecuador.

Johannisson,B. (2016). Document details - Limits to and prospects of entrepreneurs hip education in the academic context. Entrepreneurship and Regional Develo pment. 28 (5-6), 403-423. http://dx.doi.org/10.1080/08985626.2016.1177982

Karimi, S., Biemans, H.J.A., Lans, T., Chizari, M., Mulder, M. 2016, The Impact of Entrepreneurship Education: A Study of Iranian Students' Entrepreneurial Intentions and Opportunity Identification. Journal of Small Business Management. 54 (1), 187-209. https://doi.org/10.1111/jsbm.12137

Mandel, R., Noyes, E. (2016). Survey of experiential entrepreneurship education offerings among top undergraduate entrepreneurship prorams. Education and Training. 58 (2), 164-178. https://doi.org/10.1108/ET06-2014-0067

Maresch, D., Harms, R., Kailer, N., Wimmer-Wurm, B. (2016), The impact of entrepreneurship education on the entrepreneurial intention of students in science and engineering versus business studies university programs. Technological Forecasting and Social Change. 104 (5), 172-179. https://doi.org/10.1016/j.techfore.2015.11.006

Martinez, F., Bajaña, J., Chavez, C., Guerrero, M., \& Oña , B. (28 de 09 de 2016). Ecosistema del emprendimiento en la universidad contemporánea. Dialnet. Didasc@lia: Didáctica y Educación, 7(6), 249-262. Obtenido de https://dialnet.unirioja.es/servlet/articulo?codigo $=6672967$

Morales-Urrutia, X., Naranjo-Gaibor, A., Espinoza-Guano, M., Morales-Urrutia, D., Simbaña-Taipe, L. (2020). A Retrospective and Prospective Analysis of Social Entrepreneurship: Popular and Solidarity Economy in Ecuador, Advances in Intelligent Systems and Computing, 1327(21), 178-189, https://doi.org/10.1007/978-3-030-68083-1_14

Nabi G. (2017), The Impact of Entrepreneurship Education in Higher Education: A Systematic Review and Research Agenda. Academy of Management Learning and Education. 16 (2), 277-299. https://doi.org/10.5465/amle.2015.0026

Naciones Unidas. (5 de marzo de 2020). Noticias ONU. Recuperado el 1 de mayo de 2021, de https://news.un.org/es/story/2020/03/1470641

Jena R.K. (2020). Measuring the impact of business management Student's attitude towards entrepreneurship education on entrepreneurial intention: A case study. Computers in Human Behavior. 107, 106275 https://doi.org/10.1016/j.chb.2020.106275 
Organización de las Naciones Unidas para la Educación, la Ciencia y la Cultura. (13 de mayo de 2020). Organización de las Naciones Unidas para la Educación, la Ciencia y la Cultura. Recuperado el 30 de abril de 2021, de Educación superior para todas las personas: https://acortar.link/dWeqFF

Organización Mundial de la Salud. (29 de junio de 2020). Organización Mundial de la Salud. Recuperado el 30 de abril de 2021, de https://www.who.int/es/news/item/29-06-2020-covidtimeline

Paternina, J. (2018). Emprendimiento escolar, una estrategia de identificación de talentos excepcionales. Revista espacios. $39 \quad$ (49). 5. http://www.revistaespacios.com/a18v39n49/a18v39n49p05.pdf

Premand, P.; Brodmann, S. A, meida, R., Grun, R., \& Barouni, M. I. (2015). Entrepreneurship Education and Entry into Self-

Employment Among University Graduates. World Development. 77 (01), 311327 https://doi.org/10.1016/j.worlddev.2015.08.028

Purzer, S., Fila, N., Nataraja, K. (2016). Evaluation of current assessment methods in $\begin{array}{lllll}\text { engineering entrepreneurship } & \text { education. } & 5 & \text { (1), }\end{array}$ https://files.eric.ed.gov/fulltext/EJ1090526.pdf

Salguero, N., García, C., Chiluisa, M. \& Castro, S. (2017). Emprendimiento, Universidad y Sociedad. Boletín Redipe. Dialnet, 6-4, 8. Obtenido de https://dialnet.unirioja.es/servlet/articulo?codigo=6145625

Secretaría de Educación Superior, Ciencia, Tecnología e Innovación (SENESCYT). (2019). Oferta académica de las Instituciones de Educaicón Superior. Recuperado el 10 de mayo de 2021, de http://admision.senescyt.gob.ec/media/2019/07/Oferta-2do-Semestre2019_Digital_.pdf

Utami, C.W. (2017). Attitude, Subjective Norms, Perceived behavior, Entrepreneurship education and Self-efficacy toward entrepreneurial intention University student in Indonesia. European Research Studies Journal. 20 (2), 475-495. https:// doi.org/10.35808/ersj/654

Valencia-Gonzalez, E., Becerra-Sarmiento, M., \& Revelo-Oña, R. (2021). Principales competencias de los emprendedores, caso Ecuador. 593 Digital Publisher CEIT, 6(5), 346-353. https://doi.org/10.33386/593dp.2021.5.683

Vásquez, C. (2017). Educación para el emprendimiento en la Universidad. Estudios de la Gestión. Revista Internacional de Administración, 27. Obtenido de https://repositorio.uasb.edu.ec/bitstream/10644/5957/1/07-ES-Vasquez.pdf

Westhead, P., Solesvik, M.Z. (2016), Entrepreneurship education and entrepreneurial intention: Do female students benefit?. International Small Business Journal: Researching Entrepreneurship. $34 \quad$ 979-1003. https://doi.org/10.1177/0266242615612534 